\title{
Advanced Observations of Lunar Physical Librations and Gravitational Fields in Japanese Lunar Missions in the Near Future
}

Hideo Hanada, Kosuke Heki, Nobuyuki Kawano, Masatugu Ooe,

Tsuneya Tsubokawa, Seiitsu Tsuruta, Toshiaki Ishikawa, Hiroshi Araki, Koji Matsumoto,

National Astronomical Observatory, 2-12 Hoshigaoka, Mizusawa, Iwate 023-0861, Japan

Takashi Takanezawa, Yusuke Kono,

Graduate University for Advanced Studies, 2-12 Hoshigaoka, Mizusawa, Iwate 023-0861, Japan

Hiroshi Karoji,

National Astronomical Observatory, 2-21-1 Osawa, Mitaka, Tokyo 181-8588, Japan

Takahiro Iwata, Yutaka Kaneko and Takaaki Yokoyama

National Space Development Agency of Japan, 2-1-1 Sengen, Tsukuba, Ibaraki 305-8505, Japan

Abstract. In the SELenological and ENgineering Explorer (SELENE) project which is the Japanese lunar program to be launched in 2003 by the Institute of Space and Astronautical Science (ISAS) and National Space Development Agency of Japan (NASDA), we measure angular distance between a radio transmitter on a relay satellite, that on the Moon and quasars by differential VLBI and determine amplitudes of the physical librations, gravitational harmonic coefficients of the Moon and lunar ephemeris with an accuracy one or two orders higher than before in cooperation with 4-way Doppler measurements and two-way Doppler and ranging measurements using the lunar orbiter and the relay satellite. We are proposing another selenodetic mission, In situ Lunar Orientation Measurement (ILOM) to study lunar rotational dynamics by direct observations of the lunar physical libration from the lunar surface with an accuracy of 1 milliarcsecond in a post-SELENE project which will be launched about three years after SELENE. Year-long trajectories of the stars provide information on various components of the physical librations and we will also try to detect the lunar free librations in order to investigate the lunar mantle and the liquid core. 


\section{Introduction}

In the long history of the observations and investigations of the motion of the Earth's axis of rotation, combination of many kinds of observation techniques including oceanic and meteorological as well as astronomical and space geodetic ones gave rise to many new findings related to Earth's structure and mechanism. The motions of the axis of rotation are divided into two groups according to the axis motion. One is the nutation or precession, which is the motion of the axis of rotation with respect to the inertial reference frame, and the other is polar motion which is the motion of the axis of rotation with respect to the frame fixed to the Earth. We call them, including variation of angular velocity around the axis, or length-of-day variation, Earth rotation fluctuations.

The Moon, on the other hand, also has rotational fluctuations corresponding to the Earth's ones which are called by the name of librations in general. There are physical and free librations of the Moon and each libration has three components. The physical librations are caused by an attraction between the moon and the planets such as the Earth and the Sun. The free librations, on the other hand, are considered to be caused by impacts of meteorites or asteroids although it has never been firmly identified by past observations. We can give constraints upon a density structure of the lunar interior from the moment of inertia of the Moon which is derived by combining the amplitudes of the physical librations and the low degree harmonics of the lunar gravitational field. In addition to this, the periods and the amplitudes of the free librations if they are present can give information related to conditions of the lunar core and the core-mantle boundary as well as the elastic properties.

There have been Lunar Laser Ranging (LLR) observations for more than 25 years and they contributed to improve the lunar libration models including the free librations (Dickey et al. 1994, Newhall and Williams 1997). LLR can determine the parameters of the physical librations with an accuracy of about $10^{-4}$ from the ranging accuracy of a few centimeters. However, it is not enough to estimate the lunar parameters by LLR observations only since LLR has no sensitivity in the direction perpendicular to the Earth-Moon direction, or it has less sensitivity for observations of the libration around the axis of the EarthMoon direction. Concerning the free librations, the amplitudes obtained from the LLR data are not accurate enough to study the lunar interior although they are comparable to those theoretically predicted by Sekiguchi $(1969,1970)$ supposing the excitation by impacts.

SELENE (SELenological and ENgineering Explorer) is the first ISAS (the Institute of Space and Astronautical Science) and NASDA (National Space Development Agency of Japan) joint project consisting of a lunar orbiter with a propulsion module, which makes a landing after a one year mission, and a relay satellite launched by H-IIA rocket in 2003 to obtain scientific data for investigations of the lunar origin and evolution and developing the technology for future lunar exploration (Sasaki et al. 1998). Differential VLBI is one of the distinctive geodetic methods in SELENE which determines the amplitude and the phase of the physical librations and the spherical harmonics of the lunar gravitational field by measuring angular distances between a radio source on the relay satellite, that on the propulsion module on the Moon, and quasers. 
We are proposing another selenodetic mission, In-situ Lunar Orientation Measurement (ILOM) to study lunar rotational dynamics by direct observations of the lunar physical libration from the lunar surface in a post-SELENE project which will be launched about three years after SELENE. They will tell us about the dynamic flattening, Love number, energy dissipation in the lunar mantle and the liquid core as well as improved value of the lunar moment of inertia.

\section{Determination of the Lunar Gravity Fields and the Physical Li- brations in SELENE Project}

It is important to know a more accurate value of the lunar moment of inertia through measurements of the second degree spherical harmonics of the lunar gravitational fields and the amplitudes of the physical librations in order to solve the important problems of the origin and the evolution of the Moon. Differential VLBI can measure the angular distance between two radio sources which are close to each other with higher sensitivity by canceling the effect of excess path delays in the ionosphere and the troposphere which are almost equal for each radio source (Counselman III et al. 1973). A VLBI network with $2000 \mathrm{~km}$ baseline can determine a phase delay with accuracy of $3.3 \mathrm{ps}$ if we measure the phase of carrier waves in X-band $(8.4 \mathrm{GHz})$ with 0.17 rad. (10 degrees) sensitivity, which is equivalent to the accuracy in angular distance of $5.3 \times 10^{-10}$ $\left(1.1 \times 10^{-4}\right.$ arcsec $)$ or is equivalent to the accuracy in position on the Moon of about $20 \mathrm{~cm}$ through the relation

$$
\Delta d=c \sigma_{\phi} A /(2 \pi f D)
$$

where $\Delta d$ is accuracy in position of a radio source on the Moon, $c$ light velocity, $\sigma_{\phi}$ error for phase delay measurements, $A$ distance between the Earth and the Moon, $f$ frequency of the continuous wave and $D$ baseline length (Kawano et al. 1994). The accuracy will be $5 \mathrm{~cm}$ if we measure the phase with $0.034 \mathrm{rad}$. (2 degrees). Differential VLBI can contribute to improvement of the accuracy of the spherical harmonics of the lunar gravitational fields in cooperation with 4-way Doppler measurements and two-way Doppler and ranging measurements by using the lunar orbiter and the relay satellite (Namiki et al. 1999).

The amplitudes of the physical librations are proportional to dynamic flattenings $\alpha, \beta$ and $\gamma$ which are respectively defined as $\alpha=(C-B) / A, \beta=$ $(C-A) / B, \gamma=(B-A) / C$ with lunar principal moments of inertia $A<B<C$ (Eckhardt 1981). The second degree gravitational harmonics are also necessary to estimate the moment of inertia of the Moon since there are relations,

$$
C / M R^{2}=4 C_{22} / \gamma
$$

or

$$
\frac{I}{M R^{2}}=\frac{4 C_{22}(3+\beta+\gamma-\beta \gamma)}{3 \gamma(1+\beta)}
$$

where $I(I=(A+B+C) / 3)$ is the mean moment of inertia (Ferrari et al. 1980). The ratio of the sensitivity of differential VLBI to the amplitude of the observed periodicity attains about $5 \times 10^{-5}$, since the sensitivity of the differential VLBI is about $5 \mathrm{~cm}$ and the libration with amplitude of 100 arcsec causes the maximum 


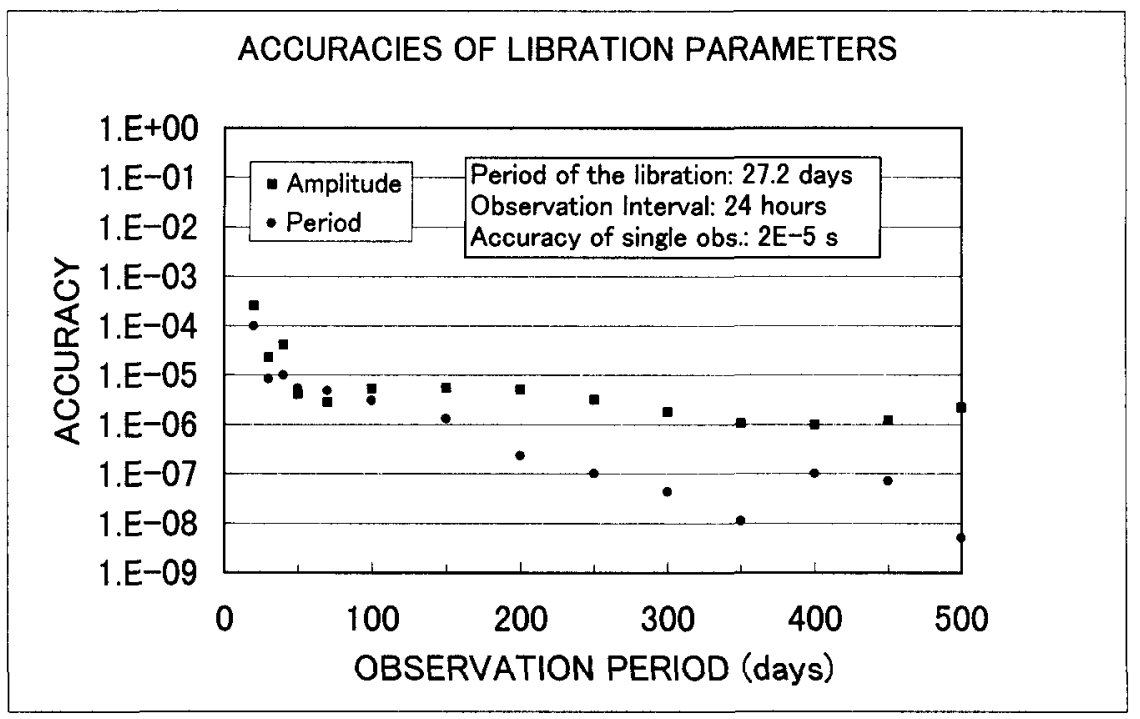

Figure 1. Relations between observation period and measurement accuracy of the amplitude and the period of the physical librations

change by about $2.6 \times 10^{-6}(0.54$ arcsec or $1 \mathrm{~km}$ on the Moon $)$ in the angular distance between the radio source on the Moon and the center of gravity of the moon which is a focus of the orbit of the relay satellite (Hanada et al., 1993). Simulations show that the VLBI observations at 24-hour intervals for longer than two months, which is the maximum lifetime of batteries for the radio source on the Moon in a certain condition (Hanada et al. 1999), can determine amplitudes of the physical librations with an accuracy of $10^{-5}$ (Figure 1).

Distinctive missions in the SELENE project are the determination of the lunar gravity field by using the differential VLBI in cooperation with 4-way Doppler measurements and two-way Doppler and ranging measurements of the lunar orbiter and the relay satellite. We can estimate the lunar gravity fields by using three dimensional tracking data, two are from the differential VLBI and one from the Doppler measurements, for longer than one year without maneuvering, and can estimate the lunar farside gravity fields by direct measurements of the orbiter passing across the farside by 4 -way Doppler tracking through the relay satellite. These new observations will improve the lunar gravity model, especially the low degree harmonics of the lunar gravitational fields, by nearly 10 times. (Namiki et al. 1999).

\section{In Situ Lunar Orientation Measurement}

The Dynamics of the Moon has been investigated mainly with the data obtained by orbiters revolving around the Moon or by laser ranging from the Earth. Many satellites have used many kinds of orbits in order to determine the lunar gravity 
field except for the farside. LLR continued observations for longer than 25 years in order to determine amplitudes and periods of the physical and the free librations, ephemeris of the Moon and also the Earth rotation parameters. As to the librations, LLR contributed largely to improvement of the physical libration model and showed the possibility of an existence of free librations. However it will be difficult to further improve the model especially of the free librations even if LLR would continue operations for another 25 years. It is not essential to increase the number of data by continuing the observations for a long time but it is important to improve the accuracy of a single measurement in order to detect and investigate damped motions such as the free librations.

We are proposing another selenodetic mission, In situ Lunar Orientation Measurement (ILOM) to study lunar rotational dynamics by direct observations of the lunar physical libration from the lunar surface in a post-SELENE project. The main component is a compact PZT telescope deployed near the lunar pole. The PZT is suitable for a positioning telescope on the Moon since a mercury pool set at the middle point of the focal length compensates the tilt of the telescope caused by thermal expansion. It is fixed at the celestial pole, and equipped with a CCD camera with $4,000 \times 4,000$ pixels to determine the instantaneous position of several tens of stars in the field of view accurate to 1 milliarcsecond (1 mas). Although a longer focal length gives rise to more accurate observation, we are compelled to adopt a focal length of $2 \mathrm{~m}$ owing to limitations of the payload. We defocus a star image by vertically displacing the CCD surface and determine the center of the star image by fitting to a Gaussian distribution model in order to compensate for the lack of focal length. Simulations show that the positioning error in the CCD plane is less than 1/1,000 of a pixel size in the condition that an exposure time is $100 \mathrm{~s}$, a pixel size is $9 \mu \mathrm{m}$, the magnitude of the star is brighter than 15 and the star image distributes over a few pixels squares. Specifications of the PZT are listed in Table 1.

Table 1. Specifications of the PZT.

\begin{tabular}{ll}
\hline \hline Caliber & $0.2 \mathrm{~m}$ \\
Focal Length & $2 \mathrm{~m}$ \\
Type & PZT \\
Resolving Power & $1^{\prime \prime}(\lambda=500 \mathrm{~nm})$ \\
Detector & $\mathrm{CCD}$ \\
Dimension of a pixel & $9 \mu \mathrm{m} \times 9 \mu \mathrm{m}\left(0.8^{\prime \prime} \times 0.8^{\prime \prime}\right)$ \\
Number of Pixels in Array & $4,096 \times 4,096$ \\
Field of View & $1^{\circ} \times 1^{\circ}$ \\
Integration Time & $<100 \mathrm{~s}$ \\
Magnitude of Target Stars & $M<13$ \\
Final Expected Accuracy & 0.001 of a pixel size (1 mas) \\
\hline
\end{tabular}

The PZT on the Moon is similar to that used for the international latitude observations of the Earth except for the half mirror above the objective, CCD, the heater beneath the mercury pool and the multiple layer insulators (MLI) around the telescope (see Figure 2). Optical energy passing through the $20 \mathrm{~cm}$ 


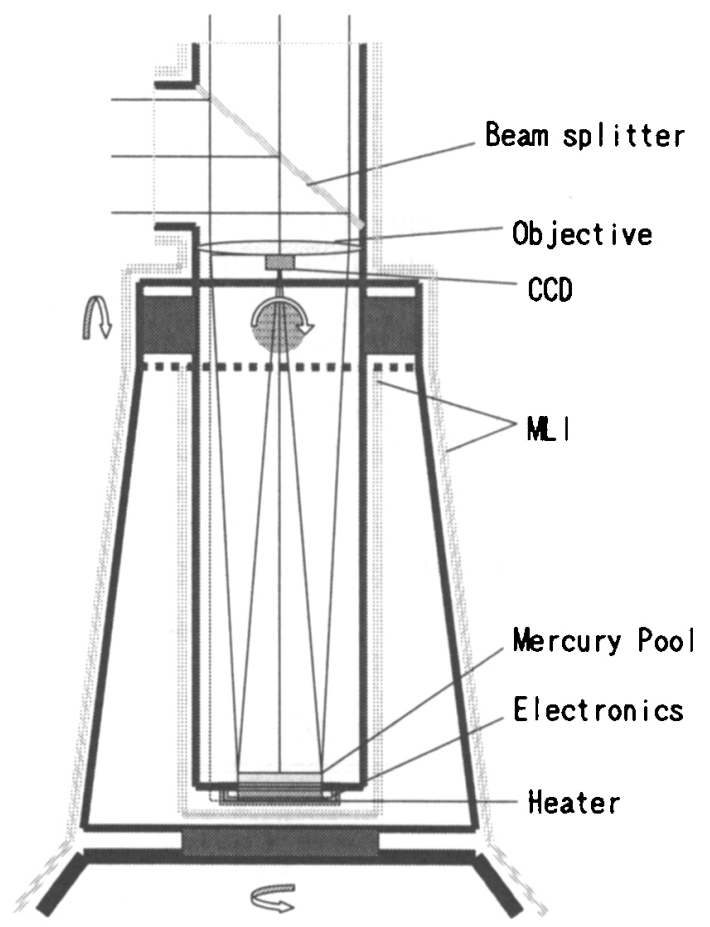

Figure 2. A schematic diagram of the PZT on the Moon. 
aperture from a star with a magnitude $M=0$ is $3.64 \times 10^{-11} \mathrm{Js}^{-1} \mathrm{~m}^{-2} \mathrm{~nm}^{-1}$ at a wavelength of $\lambda=550 \mathrm{~nm}$. A CCD having a bandwidth of $600 \mathrm{~nm}$ with a center frequency of $550 \mathrm{~nm}$ can receive the optical energy of $2.75 \times 10^{-9} \mathrm{Js}^{-1}$, which is equivalent to number of photons of about $7.6 \times 10^{9}$ since a photon has energy of $h \nu=3.61 \times 10^{-19} \mathrm{~J}$ where $h$ is the Planck's constant $\left(h=6.624 \times 10^{-34} \mathrm{Js}\right)$. A pixel receives about $7.6 \times 10^{7}$ photons in average if the star image is expanded over $10 \times 10$ pixels. Then, the pixel can convert the optical energy to about $3.42 \times 10^{7}$ electrons if the quantum efficiency is $45 \%$. Stars which generate the electrons of number from $2 \times 10^{2}$ to $4 \times 10^{7}$ are necessary in order that the signal to noise ratio should be larger than 10 since the typical dark current in the pixel is 20 electrons/s, the saturated current is $4 \times 10^{7}$ electrons/s. This leads to the optimum magnitudes from $M=-0.2$ to 13.1 from a relation between the magnitude $(n, m)$ and the optical energy $\left(L_{n}, L_{m}\right)$ as

$$
n-m=\frac{1}{0.4} \log \frac{L_{m}}{L_{n}}
$$

In principle, star position on the focal plane does not change even if the telescope is inclined a little. However, the tilt of the telescope affects the star position due to aberrations of the objective in the actual case. We investigated the relation between the deviation of the star position on the CCD and the tilt of the telescope for a ray passing through the center of the objective, which is composed of one convex lens for simplicity in this case, by a ray tracing method and found that the effect of a tilt of less than 100 arc seconds upon the star position does not exceed 1 mas.

There are many problems which must be solved before the project will start. Optical design of the PZT objective which has no spherical aberration, no chromatic aberration and no other aberrations exceeding 1 mas, design of a mercury pool whose surface has no distortion causing a deviated ray exceeding 1 mas are important problems. We have started feasibility studies and basic experiments of the PZT in order to realize the project.

\section{Conclusion}

In the SELENE project, we will determine amplitudes of the physical librations, gravitational harmonic coefficients of the Moon and lunar ephemeris with an accuracy one or two orders higher than currently available by combination of VLBI measurements of radio transmitters on and around the Moon and the 4-way Doppler measurements and two-way Doppler and ranging measurements by using the lunar orbiter and the relay satellite. We are proposing another selenodetic mission as a post-SELENE project to study lunar rotational dynamics by direct observations of the lunar physical and the free libration from the lunar surface. A PZT telescope equipped with a CCD camera which will observe instantaneous positions of several tens of stars near the lunar pole with 1 milli-arc-second accuracy is under investigation. 


\section{References}

Counselman III, C. C., Hinteregger, H. F., King, R. W. and Shapiro, I. I. 1973, Science, 181, 772.

Dickey, J. O., Bender, P. L., Faller, J. E., Newhall, X X, Ricklefs, R. L., Ries, J. G., Shelus, P. J., Veillet, C., Whipple, A. L., Wiant, J. R., Williams, J. G. and Yoder, C. F. 1994, Science, 265, 482.

Eckhardt, D. H. 1981, The Moon and the Planets, 25, 3.

Ferrari, A. J., Sinclair, W. S., Sjogren, W. L., Williams, J. G. and Yoder, C. F. 1980, J. Geophys. Res., 85, 3939.

Hanada, H., Ooe, M., Kawaguchi, N., Kawano, N., Kuji, S., Sasao, T., Tsuruta, S., Fujishita, M. and Morimoto, M., 1993, J. Geomag. Geoelectr., 45, 1405.

H. Hanada, Iwata, T., Kawano, N., Heki, K., Tsuruta, S., Ishikawa, T., Araki, H., Matsumoto, K., Kono, Y., Kaneko, Y., Ogawa, M., Iijima, Y., Koyama, Y., Hosokawa, M., Miyazaki, T., Namiki, N., Sengoku, A., Fukuzaki, Y., Ikeda, T., Fuke, F. and RISE group, 1999, Proc. Int. Workshop. Geodetic Measurements by the Collocation of Space Techniques on Earth (GEMSTONE), 126.

Kawano, N., Kuji, S., Tsuruta, S., Sato, K.-H., Iwadate, K., Hanada, H., Ooe, M., Kawaguchi, N., Mizutani, H. and Fujimura, A., 1994, J. Jap. Soc. Planet. Sci., 3, 159.

Namiki, N., Hanada, H., Tsubokawa, T., Kawano, N., Ooe, M., Heki, K., Iwata, T., Ogawa, M., Takano, T. and RSAT/VRAD/LALT mission group, 1999, Adv. Space Res., 23, 1817.

Newhall, X X and Williams, J. G. 1997, Celestial Mechanics and Dynamical Astronomy, 66, 21.

Sasaki, S., Tijima, Y., Kato, M., Hashimoto, M., Mizutani, H., Tsuruta, K. and Takizawa, Y. 1998, Proc. 21st Inter. Symp. Space Tech. Sci., 98-i-01.

Sekiguchi, N. 1969, Moon, 2, 78.

Sekiguchi, N. 1970, Moon, 2, 136. 